\title{
Multiple Plantar Poromas in a Stem Cell Transplant Patient
}

\author{
Rachel L. Marsh ${ }^{1}$, Benjamin Kaffenberger ${ }^{2}$, Llana Pootrakul ${ }^{2}$, Catherine Chung ${ }^{3}$ \\ 1. Dermatology, The Ohio State University College of Medicine, Columbus, USA 2. Dermatology, The Ohio State \\ University Wexner Medical Center, Columbus, USA 3. Division of Dermatology and Dermatopathology, The Ohio State \\ University Wexner Medical Center, Columbus, USA
}

Corresponding author: Catherine Chung, catherine.chung@osumc.edu

\begin{abstract}
Poromatosis, or the formation of multiple eccrine poromas, is associated with chronic immunosuppression, lymphoproliferative neoplasms, and stem cell transplantation, though the etiology and clinical significance remain poorly understood. Eccrine poromas are asymptomatic, may appear years after treatment, and overlap morphologically with other diagnoses, particularly human papillomavirus-associated verrucae, to which immunosuppressed patients may be predisposed and commonly occur in similar sites. We report a 47year-old female on chronic immunosuppression who developed multiple plantar eccrine poromas three years after achieving acute myeloid leukemia (AML) remission following treatment with chemotherapy, total body irradiation, and allogenic stem cell transplantation. We propose that early recognition, timely treatment, and regular follow-up skin examinations are necessary in the setting of multiple poromas to reduce the risk of malignancy and avoid delays in diagnosis.
\end{abstract}

Received 02/03/2020 Review began 02/19/2020 Review ended 06/16/2020 Published 06/22/2020

(c) Copyright 2020

Marsh et al. This is an open access article distributed under the terms of the Creative Commons Attribution License CC-BY 4.0., which permits unrestricted use, distribution, and reproduction in any medium, provided the original author and source are credited.
Categories: Dermatology

Keywords: eccrine poroma, poromatosis, multiple poromas, stem cell transplantation, acute myeloid leukemia, lymphoproliferative neoplasm, graft versus host disease, chronic immunosuppression

\section{Introduction}

Poromas, benign adnexal tumors composed of apical glandular ductal cells with presumed eccrine origin, are commonly present in middle-aged adults as asymptomatic slow-growing solitary lesions. They are characterized by smooth or verrucous flesh-colored papules, nodules, or plaques with acral predilection, presumably due to high eccrine sweat gland density [1]. However, recent evidence suggests the scalp, trunk, and extremities are involved more frequently than previously believed [2]. Diagnostic histologic features include broad anastomosing bands of cuboidal-shaped epithelial cells with monomorphous nuclei and minimal cytologic atypia [3]. Rarely, patients develop grouped or widespread multiple eccrine poromas, known as eccrine poromatosis. Poromatosis has previously been associated with lymphoproliferative neoplasms and stem cell transplantation, though the etiology and clinical significance remain poorly understood. This report adds to the limited literature of poromatosis in transplant patients with a history of hematologic malignancy.

\section{Case Presentation}

A 47-year-old African American female presented with a rapidly growing, tender lesion on the left plantar foot. The patient was diagnosed with acute myeloid leukemia (AML) three years prior to presentation and was treated with high dose cytarabine, daunorubicin, intrathecal methotrexate, and total body irradiation followed by an allogenic stem cell transplant. She achieved remission, but her post-transplantation course was complicated by multisystem graft-versus-host disease (GVHD) requiring long-term immunosuppressive therapy. Examination of the feet revealed a $1 \mathrm{~cm}$ verrucous plaque on the left plantar surface with an adjacent $0.5 \mathrm{~cm}$ papule and two similar-appearing lesions on the right plantar foot (Figure 1 ). 


\section{Cureus}

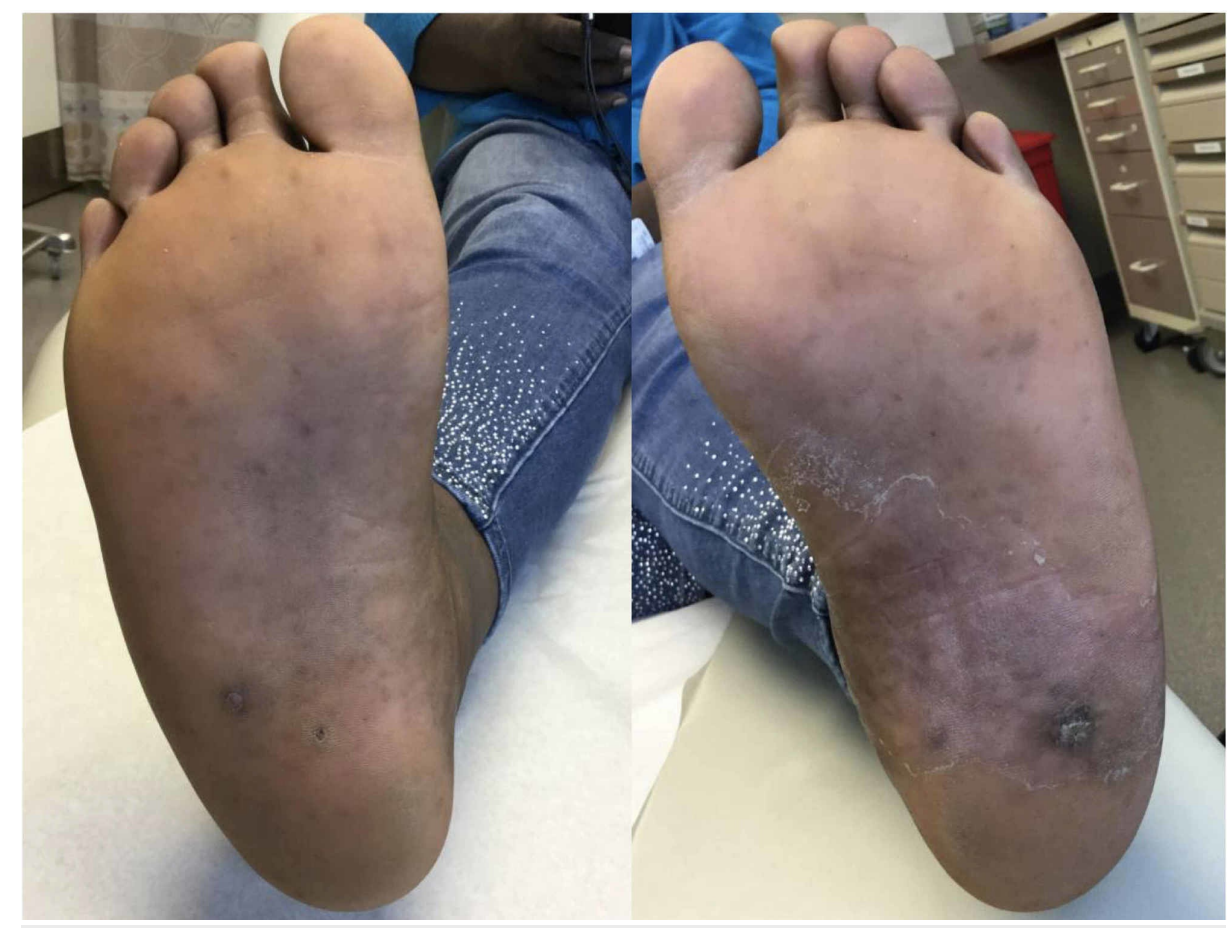

FIGURE 1: Multiple verrucous and hyperkeratotic lesions on the bilateral plantar feet.

The clinical impression was verruca, but given the patient's immunocompromised status, shave biopsy was performed on the left plantar foot to exclude malignancy. The lesion demonstrated epidermal papillomatosis with hyperkeratosis and intraepidermal proliferation of monomorphous ovoid cells with formation of ductal spaces, consistent with intraepidermal poroma (Figure 2). Excisional biopsies were then performed on remaining lesions, demonstrating similar histopathologic findings. Lesions did not recur after 15 months of serial follow-up skin examinations.

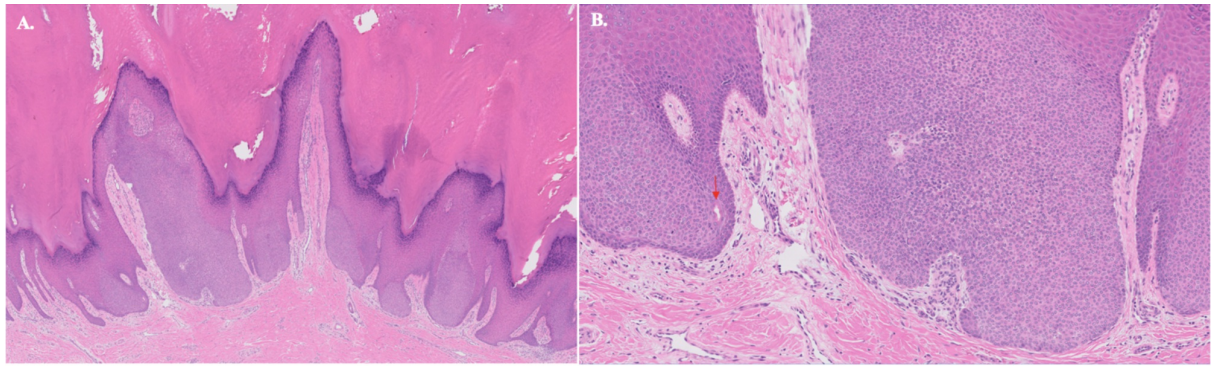

\section{FIGURE 2: Histopathologic findings of plantar eccrine poroma.}

A. Low-power magnification demonstrates papillomatosis, hyperkeratosis, and an intraepidermal proliferation of pale-staining squamoid cells (H\&E 5x). B. Higher magnification reveals a monomorphous proliferation of ovoid cells with duct formation (arrow; H\&E 20x). All lesions sampled demonstrated similar histopathologic findings.

\section{Discussion}

Multiple poromas have been reported in patients with a history of lymphoproliferative neoplastic conditions treated with radiation therapy, chemotherapy, and stem cell transplantation [4-12]. Importantly, onset of multiple poromas involving acral and nonacral sites can vary from months to years after treatment cessation. Because solitary poromas can develop at sites of trauma, it is hypothesized radiotherapy and chemotherapy may facilitate poromatosis through cytotoxic-mediated damage and subclinical injury in the eccrine glands [8]. Remodeling or regeneration of damaged eccrine apparatuses may explain long latency periods between treatment cessation and the development of multiple poromas [5]. Chronically immunosuppressed patients appear at higher risk for poromatosis. There are several reports of multiple poromas developing in patients with GVHD on chronic immunosuppression following allogenic stem cell or 
bone marrow transplants [4, 7]. Human papillomavirus (HPV) infection in immunosuppressed patients may further potentiate the development of multiple eccrine poromas [4].

Data on longitudinal outcomes and evidence-based treatment guidelines are lacking in patients with multiple poromas. Solitary poromas carry a favorable prognosis, and treatment with surgical excision or electrosurgical destruction is curative. However, it is estimated that $18 \%$ of longstanding solitary eccrine poromas may progressively degenerate into malignant porocarcinoma [13]. Porocarcinomas have a propensity to metastasize through lymphatic spread, and thus require prompt treatment with wide local excision or Mohs micrographic surgery. Individuals with a history of immunosuppression, exposure to chemical agents, and chronic ultraviolet exposure are at increased risk for porocarcinoma development [14]. These risk factors overlap with factors associated with eccrine poromatosis, suggesting patients with multiple poromas may have a higher risk of developing porocarcinoma. However, there remains a paucity of data on malignant transformation rates and de novo porocarcinoma formation in the context of eccrine poromatosis. While more data are needed to elucidate long-term clinical outcomes, we propose that serial follow-up full body skin examinations are necessary to mitigate risk of malignancy in patients with poromatosis.

\section{Conclusions}

In summary, we report a 47-year-old female in AML remission on chronic immunosuppressive therapy who developed multiple plantar eccrine poromas. Eccrine poromas overlap morphologically with HPV-associated verrucae, to which these patients may be predisposed and commonly occur in similar sites. To facilitate early recognition and avoid delays in diagnosis, clinicians should consider eccrine poromas in the context of pertinent history and physical exam findings. Further study is needed to clarify mechanisms facilitating poromatosis and enhance guidelines for appropriate therapeutic management.

\section{Additional Information \\ Disclosures}

Human subjects: Consent was obtained by all participants in this study. Conflicts of interest: In compliance with the ICMJE uniform disclosure form, all authors declare the following: Payment/services info: All authors have declared that no financial support was received from any organization for the submitted work. Financial relationships: All authors have declared that they have no financial relationships at present or within the previous three years with any organizations that might have an interest in the submitted work. Other relationships: All authors have declared that there are no other relationships or activities that could appear to have influenced the submitted work.

\section{References}

1. Su P, Heng JK, Chen Wee Aw D, Tan KB: Characteristics of eccrine tumors in a tertiary institution: a 5-year retrospective study. Skinmed. 2016, 14:175-180.

2. Betti R, Bombonato C, Cerri A, Moneghini L, Menni S: Unusual sites for poromas are not very unusual: a survey of 101 cases. Clin Exp Dermatol. 2014, 39:119-122. 10.1111/ced.12185

3. McCalmont T, Pincus LB: Adnexal neoplasms. Dermatology. Bolognia, J, Schaffer JV, Cerroni L (ed): Elevsier, China; 2018. 2:1945.

4. Diamantis ML, Richmond HM, Rady PL, Tyring SK, Cutlan JE, Torres-Cabala C, Hymes SR: Detection of human papillomavirus in multiple eccrine poromas in a patient with chronic graft-vs-host disease and immunosuppression. Arch Dermatol. 2011, 147:120-122. 10.1001/archdermatol.2010.383

5. Fujii K, Aochi S, Takeshima C, et al.: Eccrine poromatosis associated with polychemotherapy. Acta Derm Venereol. 2012, 92:687-690. 10.2340/00015555-1279

6. Kurokawa M, Amano M, Miyaguni H, Tateyama S, Ogata K, Idemori M, Setoyama M: Eccrine poromas in a patient with mycosis fungoides treated with electron beam therapy. Br J Dermatol. 2001, 145:830-833. 10.1046/j.1365-2133.2001.04475.x

7. Mahlberg MJ, McGinnis KS, Draft KS, Fakharzadeh SS: Multiple eccrine poromas in the setting of total body irradiation and immunosuppression. J Am Acad Dermatol. 2006, 55:46-49. 10.1016/j.jaad.2006.02.052

8. Navi D, Fung M, Lynch PJ: Poromatosis: the occurrence of multiple eccrine poromas . Dermatol Online J. 2008, 14:3.

9. Nguyen BT, Lortscher DN, Lee RA: Multiple poromas in a bone marrow transplant recipient: a case report . Dermatol Online J. 2012, 18:9.

10. Ullah K, Pichler E, Fritsch P: Multiple eccrine poromas arising in chronic radiation dermatitis . Acta Derm Venereol. 1989, 69:70-73.

11. Garshick M, DeFilippis EM, Harp J, Gaan J: Eccrine poromatosis in a patient with acute myeloid leukemia following chemotherapy. Dermatol Online J. 2014, 20:11.

12. Mayo TT, Kole L, Elewski B: Eccrine poromatosis: case report, review of the literature, and treatment. Skin Appendage Disord. 2015, 1:95-98. 10.1159/000438458

13. Robson A, Greene J, Ansari N, Kim B, Seed PT, McKee PH, Calonje E: Eccrine porocarcinoma (malignant eccrine poroma): a clinicopathologic study of 69 cases. Am J Surg Pathol. 2001, 25:710-720. 10.1097/00000478-200106000-00002

14. Luz MA, Ogata DC, Montenegro MF, Biasi LJ, Ribeiro LC: Eccrine porocarcinoma (malignant eccrine poroma): a series of eight challenging cases. Clinics. 2010, 65:739-742. 10.1590/\$1807-59322010000700014 